ISSN: $2456-8686,5(1), 2021: 117-122$

https://doi.org/10.26524/cm98

ORIGINAL RESEARCH

Open Access

\title{
Fuzzy Proper Bi-Magic Labeling on Tadpole Graph
}

Bharathi T*1 and Felixia $\mathrm{S}^{2}$

Received: 21 April 2021/ Accepted: 30 May 2021/ Published online: 18 June 2021

(C)Sacred Heart Research Publications 2017

\begin{abstract}
The perception of Fuzzy Proper Bi-Magic labeling for Tadpole Graph also-ran newly derived and the properties of fuzzy bi-magic of Tadpole graph has been discussed. The property of Fuzzy Bi-Magic of Tadpole graph is the sum of the labels on the vertices and the labels of their incident edges is one of the constants either $k_{1}$ or $k_{2}$ but $k_{2}$ is unique.
\end{abstract}

Key words: Fuzzy Bi-Magic Labeling, Fuzzy Graph, Fuzzy Labeling, Fuzzy Magic Labeling, Tadpole Graph.

\section{Introduction}

Fuzzy is a recently materialize mathematical mold work to illustrate the circumstance of trouble in real life adversity. It was announced by L. A. Zadeh in 1965, placed on Zadehs fuzzy relation the early definition of a fuzzy graph was announced by Kauffman in 1973 [1]. In 2012 A. Nagoor Gani et al., announced a new perception of Fuzzy labeling [5]. A Graph is called fuzzy labeling Graph if it has fuzzy labeling [4, 5]. A. Nagoor Gani has announced fuzzy magic graph in which $\beta(u)+\beta(u v)+\beta(v)$ has a same constant value for all $u, v \in V$ [5]. Later J. Basker Babujee has announced the assumption of Bi-magic in which there endure two constants $k_{1}$ and $k_{2}[1]$.

\section{Definitions}

Definition 2.1 A Tadpole graph $T_{(r, s)}$ in a fuzzy graph is a sequence of distrinct nodes $v_{1}, v_{2}, \cdots, v_{n}$ such that $\left(v_{i}, v_{i+1}\right)>0,1 \leq i \leq n$, here $n \geq 4$ is said to be the length of the Tadpole graph $T_{(r, s)}$. The successive pairs $v_{i}, V_{i+1}$ are called the edge of the Tadpole graph. A Tadpole graph $T_{(r, s)}$ is called a cycle if $v_{1}=v_{n}$ for $n \geq 4$.

\footnotetext{
1,2 Department of Mathematics, Loyola College, University of Madras, Chennai.

Email: *,1 prith.bharu23@gmail.com, 2felixiaraj1994@gmail.com
} 
The strength of a Tadpole graph $T_{(r, s)}$ is defined as $\bigwedge_{i=1}^{n} \delta\left(v_{i}, v_{i+1}\right)$ of a graph $G$, the degree of a nodes $v$ is determined as $d(v)=\sum_{u \neq v, u \in v}^{i=1} \delta(u, v)$. The strength of connectedness [2] of a pair of nodes $\left(u_{i}, v_{j}\right) \in V$ in a fuzzy graph is defined as $C O N N_{G}\left(u_{i}, v_{j}\right)=C O N N_{G}\left(u_{i}, v_{j}\right)$ such that $C O N N_{G}\left(u_{i}, v_{j}\right)=\max \left(\mu^{\infty}\left(u_{i}, v_{j}\right)\right)$. An edge $\left(u_{i}, v_{i+1}\right)$ is caleed a fuzzy bridge [11] if its delection, decrease the strength of connectedness between some pair of vertex in a graph $G$. A node $u_{i}$ is said to be a cut node [1] if its delection, shorten the strength of connectedness between some other pair of vertex in a graph $G$.

Definition 2.2 Fuzzy Labeling: A Fuzzy graph $G=(V, \beta, \delta)$ is a threefold consisting of a nonempty set $V$ together with pair of functions $\beta: V \rightarrow[0,1]$ and $\delta: E \rightarrow[0,1]$ such that for all $u, v \in V, \delta(u, v) \leq \beta(u) \wedge \beta(v)$. A fuzzy labeling graph is said to be Fuzzy magic graph if $\beta(u)+\beta(u v)+\beta(v)$ has a same value for all $u, v \in V$ which is denoted as $M_{\circ}(G)$, a fuzzy labeling graph admits Bi-magic labeling if the sum of membership values of vertices and edges incident at the vertices are $k_{1}$ and $k_{2}$, where $k_{1}$ and $k_{2}$ are constants and denoted by $B M_{\circ}\left(T_{(r, s)}\right)$. A fuzzy labeling graph which permits a Bi-magic labeling is called a fuzzy bi-magic labeling graph. The following represents fuzzy bi-magic labeling where $k_{1}=0.16$ and $k_{2}=0.18$.

\section{Result and Discussion}

Tadpole graph $T_{(r, s)}$ : Tadpole graph $T_{(r, s)}$ is a graph obtained by joining a cycle $c_{r}, r \geq 3$ to a path $p_{s}, s \geq 1$ with a bridge.

Theorem 3.1 Every Tadpole graph $T_{(r, s)}$ is a Fuzzy proper Bi-magic graph.

Proof: Let $T_{(r, s)}$ be a graph with cycle $c_{r}, r \geq 3$ to a path $p_{s}, s \geq 1 v_{1}, v_{2}, \ldots, v_{n}$ and $v_{1} v_{2}, v_{2} v_{3}, \ldots, v_{n-1} v_{n}$ are the nodes and edges of $T_{(r, s)}$. Let $F \rightarrow(0,1]$ such that one can choose $F=0.1$ if $n \leq 4$. and $F=0.01$ if $n>4$.

If the length of the $T_{(r, s)}$ is odd then the fuzzy labeling is defined as follows,

$$
\begin{aligned}
& \beta\left(v_{2 i}\right)=(2 n+1-i)(F), 1 \leq i \leq \frac{n-1}{2} \\
& \beta\left(v_{2 i-1}\right)=\beta\left(v_{n-1}\right)-i(F), 1 \leq i \leq \frac{n+1}{2} \\
& \delta\left(v_{n}, v_{n-\left(C_{n}-i\right)}\right)=\beta\left(v_{n}\right)-i(F), 2 \leq i \leq n
\end{aligned}
$$

Case (i) i is even

Then $i=2 y$ for any positive integer $\mathrm{y}$ 
ISSN: 2456-8686, 5(1), 2021:117-122

https://doi.org/10.26524/cm98

For each edge $\left(v_{i}, v_{i+1}\right)$

$$
\begin{aligned}
B M_{0}\left(T_{(r, s)}\right) & =\beta\left(v_{i}\right)+\beta\left(v_{i}, v_{i+1}\right)+\beta\left(v_{i+1}\right) \\
& =\beta\left(v_{2 y}\right)+\beta\left(v_{2 y}, v_{2 y+1}\right)+\beta\left(v_{2 y+1}\right) \\
& =(2 n+1-y)(F)+\beta\left(v_{n}\right)-(n+1-2 y)+\beta\left(v_{n-1}\right)-(y+1)(F) \\
& =\beta\left(v_{n}\right)+\beta\left(v_{n-1}\right)+(n-1)(F)
\end{aligned}
$$

Case (ii) $\mathrm{i}$ is odd

Then $i=2 y+1$ for any positive integer $y$

For each edge $\left(v_{i}, v_{(i+1)}\right)$

$$
\begin{aligned}
B M_{0}\left(T_{(r, s)}\right) & =\beta\left(v_{i}\right)+\beta\left(v_{i}, v_{(i+1)}\right)+\beta\left(v_{(i+1)}\right) \\
& =\beta\left(v_{(2 y+1)}\right)+\beta\left(v_{(2 y+1)}, v_{(2 y+2)}\right)+\beta\left(v_{(2 y+2)}\right) \\
& =\beta\left(v_{n-1}\right)-(y+1)(F)+(2 n+1-(y+1)(F))+\beta\left(v_{n}\right)-(n-2 y) F \\
& =\beta\left(v_{(n-1)}\right)+\beta\left(v_{n}\right)+(n-1)(F)
\end{aligned}
$$

If the length of the $\left.T_{(} r, s\right)$ is even then the fuzzy labeling is defined as follows.

$\beta\left(v_{2 i-1}\right)=(2 n+1-i)(F), 1 \leq i \leq \frac{n}{2}$

$\beta\left(v_{2 i}\right)=\beta\left(v_{n-1}\right)-i(F), 1 \leq i \leq \frac{n}{2}$

$\delta\left(v_{n}, v_{n-\left(c_{n}-i\right)}\right)=\beta\left(v_{n}\right)-i F, i=1$ (where $C_{n}$ is the length of a cycle)

$\delta\left(v_{n-i+2}, v_{n-i+1}\right)=\beta\left(v_{n}\right)-i(F), 2 \leq i \leq n$

Case (iii) $\mathrm{i}$ is even

Then $i=2 y$ for any positive integer $y$

For each edge $\left(v_{i}, v_{i+1}\right)$

$$
\begin{aligned}
B M_{0}\left(T_{(r, s)}\right) & =\beta\left(v_{i}\right)+\beta\left(v_{i}, v_{i+1}\right)+\beta\left(v_{i+1}\right) \\
& =\beta\left(v_{(2 y)}\right)+\beta\left(v_{2 y}, v_{2 y+1}\right)+\beta\left(v_{2 y+1}\right) \\
& =(2 n+1-y)(F)+\beta\left(v_{n}\right)-(n+1-2 y)+\beta\left(v_{n-1}\right)-(y+1)(F) \\
& =\beta\left(v_{n}\right)+\beta\left(v_{n-1}+(n-1)\right)(F)
\end{aligned}
$$

Case (iv) $i$ is odd

Then $i=2 y+1$ for any positive integer $y$

For each edge $\left(v_{i}, v_{i+1}\right)$ 
ISSN: 2456-8686, 5(1), 2021:117-122

https://doi.org/10.26524/cm98

$$
\begin{aligned}
B M_{0}\left(T_{(r, s)}\right) & =\beta\left(v_{i}\right)+\beta\left(v_{i} \cdot v_{i+1}\right)+\beta\left(v_{i+1}\right) \\
& =\beta\left(v_{2 y+1}\right)+\beta\left(v_{2 y+1}, v_{2 y+2}\right)+\beta\left(v_{2 y+2}\right) \\
& =\beta\left(v_{n-1}\right)-(y+1)(F)+(2 n+1-(y+1)(F))+\beta\left(v_{n}\right)-(n-2 y)(F) \\
& =\beta\left(v_{n-1}\right)+\beta\left(v_{n}\right)+(n-1)(F)
\end{aligned}
$$

Case (v) $i=1$

$$
\begin{aligned}
B M_{0}\left(T_{(r, s)}\right) & =\beta\left(v_{n}\right)+\beta\left(v_{n}, v_{n-\left(c_{n}-i\right)}\right)+\beta\left(v_{n}, v_{n-\left(c_{n}-i\right)}\right) \\
& =2 n+\beta\left(v_{n-1}\right)-F+\beta\left(v_{n}\right)-F \\
& =2 n+\beta\left(v_{n-1}\right)+\beta\left(v_{n}\right)-2 F
\end{aligned}
$$

Therefore in both cases the magic value $B M_{0}(G)$ is same.

Thus $T_{(r, s)}$ is fuzzy proper bi-magic graph.

\section{Verification:}

If the length of the $T_{(r, s)}$ is even

$$
\begin{aligned}
& \beta\left(v_{1}\right)+\delta\left(v_{1}+v_{2}\right)+\beta\left(v_{2}\right)=0.29 \\
& \beta\left(v_{2}\right)+\delta\left(v_{2}+v_{3}\right)+\beta\left(v_{3}\right)=0.29 \\
& \beta\left(v_{3}\right)+\delta\left(v_{3}+v_{4}\right)+\beta\left(v_{4}\right)=0.29 \\
& \beta\left(v_{4}\right)+\delta\left(v_{4}+v_{5}\right)+\beta\left(v_{5}\right)=0.29 \\
& \beta\left(v_{5}\right)+\delta\left(v_{5}+v_{6}\right)+\beta\left(v_{6}\right)=0.29 \\
& \beta\left(v_{6}\right)+\delta\left(v_{6}+v_{7}\right)+\beta\left(v_{7}\right)=0.29 \\
& \beta\left(v_{7}\right)+\delta\left(v_{7}+v_{8}\right)+\beta\left(v_{8}\right)=0.29 \\
& \beta\left(v_{8}\right)+\delta\left(v_{8}+v_{4}\right)+\beta\left(v_{4}\right)=0.28
\end{aligned}
$$

Here $k_{1}=0.29, k_{2}=0.28$ and $k_{2}$ is unique.

\section{Remark 3.2}

1. If $\mathrm{G}$ is a Fuzzy Proper Bi-Magic Graph, then $d(u) \neq d(v)$ for each pair of vertices $u$ and $v$.

2. For any Fuzzy proper bi-magic graph, $0 \leq d_{s}(v) \neq d(v)$. 
3. Sum of the degree of all vertices in a fuzzy proper bi-magic graph is equal to two times of the sum of membership values of all edges (i.e). $\sum_{i=1}^{n} d\left(v_{i}\right)=2 \sum_{u \neq v} \delta(u, v)$.

4. Sum of strong degree of all vertices in a fuzzy proper bi-magic graph is equal to two times of the sum of the membership values of all strong edges in G (i.e) $\sum_{i=1}^{n} d_{s}\left(v_{i}\right)=2 \sum_{u \in N_{s}(v)} \delta(u, v)$.

\section{Properties of The Fuzzy Proper Bi-magic Graph}

For every Fuzzy proper bi-magic graph G, there exists at least one Fuzzy bridge. Consider the path $\left(v_{4}, v_{6}\right), \mu_{1}^{\infty}=\min \left(\delta_{2}\left(v_{4}, v_{5}\right), \delta_{2}\left(v_{5}, v_{6}\right)\right)=\min (0.04,0.05)=0.04$ Let the strength of the path $v_{4}, v_{7}, v_{6}$ be denoted as $\mu_{2}^{\infty}$

$C O N N_{G}\left(v_{4}, v_{6}\right)=\max \left(\mu_{1}^{\infty}, \mu_{2}^{\infty}\right)=0.06$

$C O N N_{G-\left(v_{4}, v_{6}\right)}\left(v_{4}, v_{6}\right)=0.06=C O N N_{G}\left(v_{4}, v_{6}\right)$

$C O N N_{G-\left(v_{4}, v_{7}\right)}\left(v_{4}, v_{7}\right)=0.04<C O N N_{G}\left(v_{4}, v_{6}\right)$

The strength of the path $\left(v_{4}, v_{6}\right)$ is denoted by $\mu^{\infty}$. Let the strength of the path $v_{4}, v_{5}, v_{6}$ be denoted as $\mu_{1}^{\infty}$, Therefore, $\left(v_{4}, v_{7}\right)$ is a Fuzzy Bridge.

Therefore, every Fuzzy proper Bi-magic labeling graph G, there ensure at least one Fuzzy Bridge. Removal of a Fuzzy cut node from a fuzzy proper bi-magic Tadpole Graph $G$, the resulting Graph $G^{*}$ also admits a fuzzy froper bi-Magic labeling if $n \geq 4$. Every fuzzy proper bi-magic graph is a fuzzy labeling graph, but converse is not true.

Proof: Consider the fuzzy proper bi-magic graph.

It satisfies the fuzzy bi-magic condition, $B M_{0}(G)=\beta(u)+\beta(u v)+\beta(v)=k_{1}$ or $k_{2}$. It also satisfies the fuzzy labeling condition, $\delta(u, v)<\beta(u) \min \beta(v)$ for all $u, v \in V$. This implies every fuzzy proper bi-magic labeling graph is a fuzzy labeling graph. Conversely, consider a fuzzy labeling graph.

It satisfies the fuzzy labeling condition, $\delta(u, v)<\beta(u) \wedge \beta(v)$ for all $u, v \in V$.

But it does not satisfies the fuzzy bi-magic labeling condition, $B M_{0}(G)=\beta(u)+\beta(u v)+\beta(v)=k_{1}$ or $k_{2}$.

As we get the magic values as $0.31,0.52,0.74,0.63$, this implies every fuzzy labeling graph is not a fuzzy proper bi-magic labeling graph.

\section{Observation:}

1. If $\mathrm{G}$ is a fuzzy proper bi-magic labeling graph, then $d(u) \neq d(v)$ for any pair of 
ISSN: 2456-8686, 5(1), 2021:117-122

https://doi.org/10.26524/cm98

nodes $u, v \in V(G)$.

2. For all fuzzy proper bi-magic tadpole graph $G$, there ensures a sub graph $G^{*}$ which is an odd number of vertices then there exists at least one pair of nodes $u$ and $v$ such that $d_{s}(u)=d_{s}(v)$.

\section{Conclusion}

The concept of fuzzy proper bi-magic labeling have been discussed. Fuzzy proper bi-magic labeling for Tadpole Graph $T_{(r, s)}$ have been newly introduced and few properties of fuzzy proper bi-magic graphs are investigated.

\section{References}

[1] Babujee, J. Baskar. Bimagic labelling in path graphs, Mathematics Education, India, 3(8), 12-16, (2004).

[2] Gani, Nagoor A, and Subahashini D R, A note on fuzzy labeling, Intern. J. Fuzzy Mathematical Archive, 4(2), 88-95 (2014).

[3] Gani, Nagoor A, and Subahashini D R, Properties of fuzzy labeling graph, Applied Mathematical Sciences, 6(70), 3461-3466 (2012).

[4] Sedlacek J, Problem 27, Theory of Graphs and its Applications, 163-164, Publ.House Czechoslovak Acad. Sci., Prague (1964).

[5] Stewart B M, Magic graphs. Canadian Journal of Mathematics 18, 1031-1059 (1966).

[6] Mathew, Sunil, John N. Mordeson, and Davender S. Malik, Fuzzy graph theory, Springer International Publishing, 2018.

[7] Trenkler M, Graphs and others combination topics, Proc. Of the 3rd Czechoskovak Symp., Prague, 1983, edited by M. Fieldler, Textezur Mathematik Bank, 59, Leipzig, 328-2001 (1983).

[8] Zadeh L A, Fuzzy sets, Information and control, 8, 338-353 (1965). 